\title{
How Do We Decide? Thought Architecture Decision Making?
}

"The important thing is to not stop questioning. Curiosity has its own reason for existing."

Albert Einstein

http://doi.org/10.21272/fmir.5(2).58-71.2021

\section{Ana Njegovanović}

Master of Economics, Lecturer at Faculty of Biotechnology in Zagreb; Faculty of Economics and Tourism, University of J. Dobrila in Pula, Croatia

\begin{abstract}
The study of decision-making is an intellectual discipline; mathematics, sociology, psychology, economics, political science, artificial intelligence, neuroscience and physics. Conventional decision theory tells us what choice of behavior should be made if we follow certain axioms. Scientific curiosity instructs us to reconsider beyond any area in which we have defined ourselves. We design the intertwining of brain, genetics, phylogenetics, and artificial and neural networks in financial trading to find the best combinations of parameter values in financial trading, incorporating them into ANN models for stock selection and trader identification. The purpose and goal of the paper is to make financial decisions in the intertwining of the brain, genetics, phylogenetics and artificial neural networks, focusing on opening new foundations, giving insights into the foundation rock that lies beneath that soil.
\end{abstract}

Science seeks basic natural laws. Mathematics seeks new theorems to build on old ones. Engineering builds systems to address human needs. The three disciplines are interdependent, but different and yet Claude Shannon simultaneously makes a central contribution to all three disciplines, this was the guiding idea of our work (finance, neuroscience, artificial intelligence)

Keywords: financial decision making, brain, genetics, phylogenetics, neural and quantum networks.

JEL Classification: A19, 016,033, G41.

Cite as: Njegovanović, A. (2021). How Do We Decide? Thought Architecture Decision Making? Financial Markets, Institutions and Risks, 5(2), 58-71. http://doi.org/10.21272/fmir.5(2).58-71.2021

Received: 25 April, 2021

Accepted: 23 May, 2021

Published: 25 June, 2021

Copyright: (C) 2021 by the author. Licensee Sumy State University, Ukraine. This article is an open access article distributed under the terms and conditions of the Creative Commons Attribution (CC BY) license (https://creativecommons.org/licenses/by/4.0/)

\section{Introduction}

Conventional decision theory only tells us which behavioral choice should be made if we follow certain axioms. However, we are motivated to periodically re-examine the questions we want to answer by looking beyond any field in which we have defined ourselves. The intertwining of genetics, phylogenetics, and artificial networks in financial trading benefits from finding the best combinations of parameter values in financial trading, by incorporating them into artificial neural network models designed to select stocks and identify traders.

Decision making is a fundamental activity of humans but also of all organisms. The phylogenetic perspective shows how decision-making mechanisms are arranged, so instead of a pyramidal structure, decisions are made by a hierarchy of cortical mechanism Thus, many different control mechanisms, neural and others, are involved in decision-making. Mutual communication is very complex, they are the result of a hierarchical network.

Today's decision makers in the fields from engineering to psychology, from medicine to economics and / or homeland security are faced with new technologies, large amounts of information to help them make good / rational decisions and the ability to share unprecedented speeds and quantities. These tools and resources should lead to better decisions. However, tools bring with them frightening new problems: huge amounts of available data are often incomplete, unreliable and / or distributed and there is great uncertainty in them; interconnected / distributed decision makers and decision-making devices must be coordinated; many data 
sources need to merge into a good decision; the exchange of information under new cooperation / competition arrangements poses security concerns.

Overcoming our natural impulses is necessary to meet the financial demands of modern society, which require us to delay gratification, save money, and build wealth (Brad Klontz, 2019.).

Money is not a neutral medium of exchange, but an extremely complex substance that has profound effects both on the human mind and on the economy as a whole. Its strength lies in the unique combination of the properties of number and the properties of things. Searle compares owning money to owning a queen in a game of chess. The letter: 'is not a matter of my having my hands on a physical object, it is rather a matter of my having certain powers of movement within a formal system... relative to other pieces. Similarly, my having a thousand dollars is not a matter of my having a wad of bills in my hand but my having certain deonti powers. I now have the right, i.e. the power, to buy things, which I would not have if I did not have the money '(Searle, 2005).

There are two contemporary theories of financial engineering related to chaos and fractals (fractal and chaos theory, branch of mathematics), which explain the duality of financial market behavior and more importantly, the way they relate to quantum finance for financial engineering in modern financial institutions. Chaos and fractals are two sides of the same coin. They show us two ways of looking at (modeling) world dynamics and all physical phenomena. Just as the particle-wave duality in quantum theory is roughly the same scenario in different (mathematical) languages. But viewed from a different perspective, chaos and fractals can, in fact, be considered a mathematical (and thus computational) way of describing particle-wave duality in terms of nonlinear equations. The path leads us to quantum finance and new decision-making architectures. With the condition of shaping high intelligence and with a greater degree of equality. "It can be achieved in at least two possible ways. One is the formation of a society of increasingly impersonal, emotionally distant people in which artificial intelligence becomes a measure of the genius of the collective. The second is the creation of a society of authentic individuals characterized by a high level of awareness, personal responsibility and integrity". (Kaku, 2014)

Today's uncertain times, from the macro level; pandemics, climate change, social and political unrest, to personal levels of job insecurity, family illnesses and various levels of social isolation - all contribute to feelings of uncertainty.

\section{How to define uncertainty?}

"Uncertainty means ambiguity, which means we have to make an effort trying to predict what will happen in preparation for facing all the different outcomes" (Donovan, 2020) In uncertainty, the brain tries to choose how to act. affect survival and reproduction". Uncertaintly is not knowing what will happen" (Mazen Kheirbek, 2020) but leads the way by combining "uncertainty" with the "threat" of anxiety. "Anxiety is an emotional response to a perceived threat that is not actually there in front of you" (Kheirbek, 2020).

"Anxiety neurons" in turn talk to the hypothalamus, a region of the brain that triggers avoidant behaviors, on a path that seems to bypass higher-order areas of the brain. The researchers showed that when the mice turned off those "anxiety neurons," they suddenly began exploring the open space, an indication that their anxiety had subsided.

How the brain can "filter" information by "learning that certain pieces of information are more important than others in order to change behavior appropriately." (Sohal, 2019).

This process, by which certain pieces of information are selectively transmitted between different parts of the brain, often involves synchronization between brain rhythms in those regions. The prefrontal cortex plays an important role in this process and can determine which information to pay attention to and which to ignore. It makes decisions based on signals from other parts of the brain, such as the hippocampus, where "anxiety neurons" reside.

Sohal's lab measured synchronization between brain rhythms in the hippocampus and prefrontal cortex during the time mice had to make decisions. Brain regions need to work together to make these decisions - to explore that open field.

Without synchronization, the brain would find it harder to decide what is important, what to focus on. Sohal's lab sees this type of problem with proper information filtering as important factors of schizophrenia, autism, and anxiety disorders. 
But Sohal reiterates that anxiety is essential. "We have to have anxiety. Otherwise, we would be doing things that are overly dangerous. Anxiety is a fundamental part of our existence", he says. "But pathological situations occur when, for whatever reason, the brain can't seem to adjust to anxiety properly, so avoid it, avoid it, avoid it."

Neural networks are revolutionizing almost all aspects of financial and investment decision making. Financial firms around the world use neural networks to solve difficult tasks that involve intuitive judgment or require the discovery of data patterns that elude conventional analytical techniques. Many observers believe that neural networks will eventually surpass both the best traders and investors. Neural networks are already used for trading in securities markets, forecasting the economy and analyzing credit risk. In fact, apart from the U.S. Department of Defense, the financial services industry has invested more money in neural network research than any other industry or government body. Unlike other types of artificial intelligence, neural networks somewhat mimic the processing characteristics of the human brain. As a result, neural networks can draw conclusions from incomplete data, recognize patterns that evolve in real time, and predict the future. I can even learn from past mistakes! In a study by Neural Networks in Finance and Investing, Robert Trippi and Efraim Turban gathered a "stellar collection" of articles by industry and academics experts on the applications of neural networks in this important arena. They discuss the successes and failures of neural networks, as well as identify the huge unrealized potential of neural networks in a number of specialized areas of financial decisionmaking. Topics include neural network basics and review, financial condition analysis, business failure prediction, debt risk assessment, security market application, and neural network approaches to financial forecasting. Nowhere else will a financial expert find such an exciting and relevant detailed examination of neural networks. Some chapters discuss how to use neural networks to predict stock markets, commodity trading, bond and mortgage risk assessment, bankruptcy prediction, and investment strategy implementation.

\section{Theoretical Framework}

\section{Brain and decision making}

Do we really make our decisions or are they made for us? And, if so, are we still responsible for them? If someone is monitoring our brain is it possible to know what action we will take first?

Experiments conducted by neuroscientist Dr. John-Dylan Haynes at the Max Planck Institute for Human Cognitive and Brain Sciences in Leipzig, in collaboration with Charité University Hospital and the Bernstein Center for Computer Neuroscience in Berlin: proves "Many processes in the brain occur automatically and without the involvement of our consciousness, "the scientists wrote on the publication of their findings". This prevents our mind from being overloaded by simple routine tasks. But when it comes to decisions, we tend to assume they are made by our conscious mind. questioned by our current findings".

However, there were also opposing beliefs. "I actually agree that the evidence points in the direction in which subconscious processes make decisions (Steven Novella)". The problem is that the fMRI studies are very tricky to do, and what you have to realize is that when you see a pretty picture of which parts of the brain are lighting up, that's a composite. You're not looking at a clear signal in one person's brain as they're doing the task. You're looking at a composite over time and over different subjects, and when you think about it, this is a binary choice - A or B - and they're looking at this little signal peaking above all this noise and coming up with 67 to 68 percent correlation. It's actually not that much better than chance. Doesn't mean it's wrong. It's just not that robust".

Decisions are the result of complex interactions between many factors. It is difficult to determine the weight of the various factors in making a final decision. Decisions made by the brain always involve many neurons.

The weight of individual neurons in the decision-making process can be reconstructed despite the interdependence between neurons (Matthias Bethge). Information enters the brain through many sensory neurons. Which neurons are crucial in passing relevant information to higher areas of the brain. A research group led by Matthias Bethge has now developed an equation that allows them to calculate the degree to which a particular sensory neuron is involved in the decision-making process.

The brain activity involved in decision choices is still not well understood. Scientists from the Okinawa Institute of Science and Technology (OIST) have identified a new area of the brain that could be involved in making cost-effectiveness decisions. 
"Previously, many neuroscientists believed that each area of the brain performs a specific function, such as facial recognition, memory, or movement" (Sieveritz, 2020), "but in more recent years, we've realized that it's far more complex, and that cognitive processes are carried out by a distributed network across the brain, with many different brain areas communicating". Understanding the functioning of cognitive decision-making abilities requires knowledge of which parts of the brain are connected to each other. These are neurons that can reach and send signals to other neurons located in different parts of the brain. However, link mapping is extremely complex. "Within each brain region, there are many different types, or sub-classes, of neurons, which you need to be able to identify, and each neuronal sub-class might only connect to one other area of the brain or be involved in one specific function. So, it's very messy and complicated". (Sierevitz, 2020).

Many neurons from the ventromedial thalamus connect to the motor cortex, other neurons reach further into the prefrontal cortex (plays a major role in more complex cognitive behaviors, including personality expression and language comprehension). "Cortico-striatal neurons in the prelimbic cortex are important for cost-benefit decision-making" (Sieveritz, 2020). If the ventromedial thalamus sends signals to these cortico-striatal neurons, it could mean that the ventromedial thalamus also plays a role in making cost-effectiveness decisions." But neurons from the ventromedial thalamus also send signals to inhibitory neurons within the prelimbic cortex. Inhibitory neurons slow or stop the firing of other neurons and are necessary to maintain brain activity under careful control. (Sieveritz, 2020).

Research is a step closer to understanding the fundamentals of the complex cost-benefit decision-making process. "Neuroscience is like a huge puzzle; anyone can only make a small piece," (Bianca Sieveritz). "But when all our research is combined, we hope to start seeing the bigger picture".

\section{Algorithms}

Automation profoundly transforms the nature of human-machine interaction. Whether and to what extent the problem arises from the use of increasingly sophisticated algorithmic decision-making tools, including those that use state-of-the-art machine learning techniques such as deep learning.

The rise of data analytics and cognitive technologies has led to an explosion in the use of algorithms for a variety of purposes, industries, and business functions. These algorithms influence decisions that have a profound impact on individuals - including the data individuals are exposed to, what jobs they offer, whether their loan applications are approved, what medical treatment is recommended by doctors, and even their treatment in the justice system.

Increasing complexity turns algorithms into "black boxes" of decision making. Objectivity and infallibility can be attributed to algorithms. Yet black boxes are vulnerable to risks (accidental or intentional biases, errors, and fraud) with the question of how to "trust" algorithmic systems.

Accepting complexity and establishing mechanisms to manage the associated risks will contribute to the effective harnessing of the power of algorithms. Companies that adapt risk-aware mindsets will have the opportunity to use market-leading algorithms, better navigate the regulatory environment, and disrupt their industries with innovation.

As algorithms become more advanced, technology experts predict that these systems will enhance human efficiency - but also compromise human autonomy, and capability? In a 2019 study by Technology Experts, 979 technology experts predict that the advancement of algorithm-driven artificial intelligence will potentially revolutionize areas such as health, education and broad aspects of the economy, the banking system. At the same time, there are concerns about the long-term impact of new tools on the essential elements of the human being.

The algorithm is based on the theory of inverse perspective in an extended incomplete additive environment of probable language preferences and its application in the selection of financial products (stocks).

The selection of financial products is one of the most important investment activities for both individuals and companies, and it is therefore very important to establish an effective and practical method for the selection of financial products. The most important investment activity for companies but also for individuals is the selection of financial products. Conducted research of the algorithm on the theory of inverse perspective (2020) showed: that a complex decision-making environment and expression habits can be helped by a language preference for choosing financial products over decision-makers' preferences. Thus, "when decision makers express their opinions by the probabilistic linguistic relationship of preferences, it is possible that the sum of 
the values of the probability information is greater than 1 . The algorithm processes the original incomplete additive probable linguistic relationship of preferences using the inverse functions of prospect theory initially. Finally, the numerical case and analysis are presented as evidence of the conclusion that the proposed algorithm is practical and robust. preference using the inverse functions of prospect theory initially. Then a model for deriving priority weights based on extended concepts is established.Finally, the numerical case and analysis are presented as evidence of the conclusion that the proposed algorithm is practical and robust".

Finally, it is necessary to emphasize the applications of the tree in financial decision-making that combines analytical approaches such as discounted cash flows (DCF) and NPV with a clear picture of decisions and events. The technique clarifies the relationship between current and future decisions and uncertain circumstances. Such information allows management to consider available courses of action with greater ease and clarity.

Although tree analysis is a challenging decision to apply when multiple sources of uncertainty are present, it still remains a widely used technique for determining the value of investment opportunities.

\section{Genetic algorithms in financial decision making}

Genetic algorithms are unique ways of solving complex problems by harnessing the power of nature. By applying these methods to securities price forecasting, traders can optimize trading rules by determining the best values to be used for each parameter for a particular security.

Genetic algorithms (GA) are methods of solving problems (or heuristics) that mimic the process of natural evolution. Genetic algorithms use natural selection concepts to determine the best solution to a problem (while artificial neural networks function like neurons in the brain).

Genetic algorithms are used as optimizers that adjust parameters to minimize or maximize some feedback measures, which can then be used alone or in the construction of the ANN.

In financial markets, genetic algorithms are most commonly used to find the best combination values of parameters in a trading rule and can be embedded in ANN models designed for stock selection and trade recognition.

The trading rule may involve the use of parameters such as moving average convergence divergence (MACD), exponential moving average (EMA) and stochastics. The genetic algorithm would then enter values into these parameters in order to maximize net profit. Over time, small changes are introduced, and those that have the desired impact are retained for the next generation.

When we combine the efficiency of nature and the speed of a computer, the financial possibilities are almost limitless. Today's traders and investment analysts are demanding faster, more elegant weapons in today's relentless financial market. Battles are now fought at computer speed, battles are fought in just a few hours. Genetic algorithms and investment strategies in clear, non-technical language reveal an extremely effective strategic decision-making process that, imaginatively used, allows traders and investment analysts to reap significant financial rewards.

Many factors can directly or indirectly affect the future cost of an investment. Such factors are often interrelated. The space of models that explain the interaction between these factors is enormous and grows exponentially with the number of given factors. Genetic programming offers an effective search engine for the space of possible models and generates rules that the user can interpret. In collaboration with the Computer Finance Research Group at the University of Essex, the application of an interactive decision-making tool has been described. EDDIE in financial decision making, including pricing and indexing applications, forecasting and arbitrage. EDDIE searches the model space using genetic programming. The candidate's solution is represented by the genetic decision tree (GDT). The basic elements of GDT are the rules and values of the forecast. For example, for a GP to function, he must be able to assess each GDT and assign it a fitness value that reflects the quality of the GDT. EDDIE maintains a set of GDTs called populations and works in iterations. In each iteration, GDTs are selected from a population: the more capable GDT is, the greater the chance that it will be selected. The set of all selected GDTs forms a pool. 


\section{Artificial neural networks}

The idea of a "thinking machine" can be traced back to the ancient Greeks. Yet our focus on knowledge of artificial neural networks is necessary for the purpose of understanding sophisticated financial markets. The complexity of ANN is reflected in the functioning, type of neural networks, and neural networks as opposed to deep learning.

Neural networks, also known as artificial neural networks (ANNs) or simulated neural networks (SNNs), are a subset of machine learning and are at the heart of deep learning algorithms. Their name and structure are inspired by the human brain, mimicking the way biological neurons signal to each other. Artificial neural networks (ANNs) consist of node layers that contain an input layer, one or more hidden layers, and an output layer. Each node or artificial neuron connects to another and has a related weight and threshold. If the output of any single node is above the specified threshold value, that node is activated and sends data to the next layer of the network. Otherwise, the data is not transferred to the next layer of the network.

Artificial neural networks inspired by human brain structures and originally developed in the academic community. Artificial neural networks learn algorithms structured as a series of interconnected layers. They offer a step-change in the power of AI and are particularly suitable for complex "deep learning" applications that require the processing of vast amounts of data and a high level of expertise and domain judgment.

These networks consist of interconnected artificial neurons. Every neuron processes the input data with a predefined mathematical function and produces and output which becomes the input for other neurons. An ANN can be divided between the input, hidden and output layers. With the given input data, the coefficients between neurons are computed to achieve accurate outputs at the end.

ANNs are a subset of machine learning models that are loosely modeled on the human brain and learn in a similar way (lots of examples). These models require very little hard coding and can perform a fairly wide range of complex tasks with relatively simple architectures (an example is AlphaGo Zeromodel, developed by Google, which has learned to play Go- a complex and challenging board game). The opinions of one of the greatest intellectuals in the world of N. Chomsky about Artificial Neural Networks are also interesting. Although ANNs are certainly not a perfect analogy for the human brain, they are nevertheless useful for highly specialized tasks, but these tasks must be sharply limited (although their range may seem vast given the memory and speed of modern computers). ANNs do not accurately model human cognition, which is so relatively rich that the involved computer systems can extend to the cellular level (Chomsky).

Artificial neural networks (ANNs) can be considered examples of mathematical innovation, providing the latest technique in the financial economist tool for modeling financial applications. The advantages are datadriven networks, especially useful for real-time critical applications that use complex data sets. ANNs can be deep or shallow and can have different structures.

Artificial Neutral Networks (ANN) allows us to mimic human thinking and thought processes in identifying optimal trading strategies in financial markets. The broader analysis includes the theory and steps involved in deriving ANN and the generic algorithm in financial markets, the accuracy of the computer learning process and appropriate ways to use that process in developing trading strategies, but also about ANN's superiority over traditional methodologies.

\section{Stock market and artificial neural networks}

The stock market is a platform for trading the company's shares and derivatives at an agreed price. Stock supply and demand drive the stock market. The stock market in any country is one of the emerging sectors. Nowadays, many people are directly or indirectly connected to this sector. Therefore, it becomes necessary to know about market trends. Thus, with the development of the stock market, people are interested in predicting stock prices. But due to the dynamic nature and rapid change in stock prices, predicting stock prices becomes a challenging task. Stock exchanges are mainly a nonparametric, nonlinear, noisy, and deterministic chaotic system.

Vector aid machines (SVMs) and artificial neural networks (ANNs) are widely used to predict stock prices and their movements. Each algorithm has its own way of learning and then prediction. An artificial neural network (ANN) is a method that involves technical analysis for forecasting in financial markets. 
The stock market is a popular investment option for investors due to the expected high returns. In contrast, stock prediction is a complex task that is accomplished with the help of artificial intelligence. This is due to stock prices that depend on many factors, including market trends and news.

Forecasting financial market time series is challenging. Such information represents high volatility in the observed data, along with the uncertainty inherent in any forecast. Therefore, wrong decisions based on such predictions can have economically catastrophic consequences for individuals, institutions and nations, as observed in the financial crisis (Podsiadlo M, Rybinski H., 2016).

According to Shi Y, Tian Y, Kou G, Peng Y, Li J., 2011), financial institutions and banks are among those branches that have relatively complete and accurate data. Typical cases include investing in stocks, predicting loan disbursements, approving loans, predicting bankruptcy, and detecting fraud. Techniques such as discriminant analysis, linear and logistic regression, overall programming, decision trees, expert systems, neural networks, and dynamic models are commonly used in financial and banking applications.

Not surprisingly, academia and the financial industry have invested a lot of time in proposing and comparing computational methods to analyze available data, in order to obtain reliable predictions. Such analyzes are used in the prevention of macroeconomic crises, forecasting stock market movements as well as for compiling investment portfolios (Podsiadlo M, Rybinski H (2016)).

Since the 1950s, as computer technology has been gradually used in commercial applications, many companies have developed databases to store and analyze the data collected. Artificial intelligence methods have been used to deal with data sets, including neural networks and decision trees. In the 1990s, the term data mining began to be used, intersecting human intervention, machine learning, mathematical modeling, and databases. Researching the theoretical components of big data or data science requires interdisciplinary efforts in mathematics, sociology, economics, computing, and management science (Shi Y, 2014).

Financial markets have a wealth of data. The question is how to effectively conduct big data analysis which is a challenge for any area. According to Xu Z, Shi Y (2015), the big data analysis process can be described by general data analysis, which consists of several steps, including data collection and management, data access and processing, data mining and interpretation, and data applications. Modeling methods can involve many techniques, and the challenge reflects when and which method is appropriate.

According to Shi Y (2014), three problems need to be addressed in order to take advantage of the use of big data in science, engineering and business applications. The first is the transformation of semi-structured and unstructured data into structured data. Others relate to complexity, uncertainty, and systematic modeling. Third, understanding the relationship of data heterogeneity, knowledge heterogeneity, and decision heterogeneity. Modeling of artificial neural network (ANN) techniques is important in this regard. It is necessary to point out that $\mathrm{ANN}$ is an artificial intelligence technique for classification, image processing and data prediction (Aziz R, Verma C, Srivastava N, 2018).

\section{Can quantum physics explain human behavior?}

Is there a link between quantum physics, computers, and financial market behavior?

The evolution of modern finance is closely related to the evolution of computers, communications, and financial mathematics. Two major changes occurred in the 1970s with the start of derivatives trading and after the 2007 crisis with the mass introduction of fintech.

Derivative prices began with the Black and Scholes equation and formulas in 1974, followed by a wealth of mathematical methods for calculating derivative prices. It should be noted that the prices of derivatives from the 1980s required supercomputers, giving large companies a great competitive advantage - before the crisis in 2007, the volume of trade was close to $\$ 1$ trillion per day. Most opinions formed the framework that derivatives enabled the completion of financial markets that enabled any cash flow.

However, there was a different course of self-regulation. Namely, the failure of Lehman Brothers, the world of finance has shown that risk exists in derivatives and that free markets cannot self-regulate. To save them, central banks injected billions of dollars, euros and yen into liquidity through quantitative easing $(\mathrm{QE})$. In the United States, the Fed has invested about $\$ 4.5$ trillion in liquidity, about one-third of the total monetary mass. 
Modern economies are shaped by complex budgets. Supercomputers are used to design products like cars and airplanes, invent new drugs, create electronic circuits, model economies, organize large-scale logistics, and study climate. However, the calculations also make it possible to make deadly weapons and, increasingly, to monitor and try to control the behavior of populations.

The importance of quantum power for finance? Namely, quantum computing would make current cryptographic techniques uncertain. Methods and algorithms will have to change. Post-quantum cryptography or quantum-resistant cryptography is a thriving sector of study both in academia and in cryptography companies. Yet major changes are in artificial intelligence (AI) and machine learning. In the areas of finance and economics, quantum computing could lead to the analysis of a large area of heterogeneous data for financing and forecasting and understanding of economic phenomena. Financial and economic data are complex and the analysis will not necessarily lead to more accurate predictions given the complexity of the data. The complexity and non-stationarity of the data can defy analysis. Can we conclude by asking the question, will the use of quantum computation reduce uncertainty?

Perhaps we could somehow draw a conclusion: Heinrich Hertz, who first created and sent radio waves, thought he was only confirming Maxwell's electromagnetic theory. Einstein never imagined that relativity could enable GPS systems. The founders of quantum mechanics never considered advances in computing or the invention of transistors. But today we are absolutely certain that the closer we get to absolute zero, the more the whole field of atomic optics and nanooptics will progress. We may one day even be able to measure quantum effects for entire human beings.

What did field psychology want and what did quantum physics field about behavior?

Do these two disciplines overlap? The difference is that one field wants to understand the fundamental nature of physical particles, while the other tries to explain human nature - along with inherent misconceptions.

"Cognitive scientists have found that there are many" irrational 'human behaviors" (Xiaochu Zhang, 2020). Classical decision theories try to predict what choices individuals will make with certain parameters, but the wrong people do not always behave as expected. Recent research shows flaws in logic and "can be well explained by quantum probability theory" (X. Zhang, 2020).

Intriguing research in the field of decision making goes in the direction of concepts borrowed from quantum mechanics that can help psychologists better predict human decision making. The scans highlighted specific areas of the brain that may be involved in quantum-like thought processes.

Just as insecurity pervades the subatomic world, it also creeps into our decision-making process, for example; we give our vote in the presidential election. Unlike classical decision theories, the quantum world leaves room for a certain degree of uncertainty.

Theories of classical psychology are based on the idea that people make decisions to maximize "rewards" and reduce "penalties" - that is, to ensure that their actions result in more positive outcomes than negative consequences. This logic, known as "consolidating learning", is consistent with Paul's (2009) conditioning, in which people learn to anticipate the consequences of their actions based on past experiences.

If they were truly constrained by this framework, people would consistently weigh the objective values of the two options before choosing between them. But in reality people don't always do that; their subjective feelings about the situation undermine their ability to make objective decisions. As well as the choice of letter or head in betting.

"It could be said that a quantum-based decision-making model basically refers to the use of quantum probability in the realm of cognition," (Emmanuel Haven and Andrei Khrennikov, 2013). Just as a particular electron might be here or there at a given moment, quantum mechanics assumes that the first toss of a coin resulted in both victory and defeat.

In the famous thought experiment, Schrödinger's cat is both alive and dead. Although caught in this ambiguous state, known as "superposition," the individual's final choice is unknown and unpredictable. Quantum mechanics also recognizes that people's beliefs about the outcome of a given decision - whether it will be good or bad - often show what their final choice is. In this way, people's beliefs interact or become "entangled" in their eventual actions. 
Human decisions and practices play an important role in many domains of human endeavor. Decision making in economics, finance, cognitive science in relation to medical roles is not structured in model building, so we conclude: decisions in medicine touch on some of the most delicate and valued aspects of us as humans, perhaps related to life and death, so structured theoretical modeling of their processes could seem almost impossible (Kuhse 1987; Brinchmann et al. 2002; Anspach 1987; Van der Maas et al. 1991; Häyry 1991). Very complicated ethical issues are associated with even simple decisions and their later medical practices, which also seems to indicate that there are only very contextual and ad hoc reasoning (Clark and Leaverton 1994; Grimes and Schulz2002; Glasser and Howard 2006). The specific situations that arise in relation to decision-making and related medical practices face aspects that are intrinsically complex, still insufficiently understood, and perhaps paradoxical, when viewed from a particular perspective. This refers to ethical issues that arise from the use of placebo controls in clinical trials (Clark and Leaverton 1994) and to other types of problems, of different natures, associated with clinical trials (Grimes and Schulz 2002; Glasser and Howard 2006). Decisions regarding clinical trials testing the same disease, which often do not converge due to inherent complex unpredictability (Glasser and Howard 2006). And finally, about the placebo effect, which indicates that there are aspects of medical practice that can themselves in an incomprehensible way affect the health or diseases of patients who have undergone them (Lasagna, 1986; de la Fuente-Fernandez et al., 2001; Diederich and Goetz, 2008; Kaptchuk et al., 2008).

"Quantum cognition", the structure is inspired by mathematical models of quantum theory (Aerts, 2009; Busemeyer and Bruza, 2012; Khrennikov, 2010; Pothos and Busemeyer, 2013; Aerts and Aerts,1995; Aerts and Sozzo, 2011). Quantum structure also plays a role in modeling human decision making in medicine. It is known that one of the main fingerprints of quantum theory is the presence of the internal and irreducible influence of the measurement procedure on the state of the entity being studied, which is often called the "observer effect" (Sassoli de Bianchi, 2018). If we think again about the general phenomenon of the placebo effect, it includes precisely this type of influence of medical practice on the health condition or disease of the patient. As an example, we can mention the unusually well-known fact that, even when patients are informed that they are being treated with placebo, the effect still remains present (Kaptchuk et al., 2008).

\section{Quantum computing - a revolution for finance}

Quantum computing is one of the most exciting emerging technologies, (Google Quantum Processor Sycamore, 2019). Quantum computing signifies a paradigm shift in computing, potentially questioning digital security and fostering scientific advances. But what exactly is quantum computing?

Let's start with classical computing. At the heart of classical computing is the concept of computer logic. Computer logic consists of information and information processing. Information is simply strings of 1 and 0. A conventional computer represents everything - from text to images - as a string of bits (binary digits). These are units 1 and 0 . Why? Well, these logic values can be represented by the state of the transistors, which are the building blocks for integrated circuits. In computers, these bits are often represented as voltage: $0 \mathrm{~V}$ means $0 ; 5 \mathrm{~V}$ means 1 . Thus, a wire with current represents essential state 1 , and a wire without current represents essential state 0 .

Information processing can be broken down into logical gates. There are 7 primary inputs (NOT, AND, OR, NAND, NOR, XOR, XNOR) that take 1 or 2 bits as input and output of one bit. Each gate performs a unique operation on the bit (s), which then determines the output. When these doors are connected by chains, you get circuits that enable the capabilities of modern computers today.

These gates consist of transistors, which in turn are made of semiconductors (like silicon). With transistors you can store any type of information and process it. The transistor can be considered a switch: if it is 'on', current flows through the wire; if 'off', there is no electricity. Modern processors have billions of transistors, and the number of transistors approximately doubles each year (this is known as Moore's Law).

Quantum computation is not as binary as classical computation. Classical computers manipulate and process bits represented by voltages; in quantum computing we use qubites (quantum bits), which are represented by electrons. Quantum computing uses the principles of quantum mechanics to process and manipulate information, namely superposition and interweaving, which provides some incredible possibilities. 
We will look at these properties from the perspective of quantum computation, not quantum mechanics. The theory behind quantum mechanics is complex and it is much easier and more relevant to understand their applications in quantum computing.

We think of bits as 01 and 0 . Bits can be anything with 2 states: true / false; On, Off; head / letter. A classic computer simply quantifies bits as integers because that's all a computer can store and process. However, Qubits are vectors. Namely, qubit represents the probability that its essential state is 0 or 1 ; instead of just 0 or 1 , they can exist as any value between 0 and 1 until measured.

When we perform certain operations on a qubit, we can force it to superposition, where it is no longer just 0 or just 1; rather it exists as a certain probability of both. These operations can be considered as the rotation of a unit vector around a Bloch sphere in a three-dimensional real vector space. A vector space is a simple space in which a vector resides. A simple two-dimensional vector lies in the vector space $\mathrm{R}^{3}$.

"When a qubit is actually measured, it will always be 0 or 1 . The act of measuring the qubit has caused the collapse of the quantum superposition, and the state of the qubit is now analogous to classical bits" in that state it will remain indefinitely. The value to which it crashes depends on its configuration, which is known as its quantum interference the qubit can return to the superposition state via quantum gates that perform an operation on one or more qubits.

More generally, the qubit state can be represented as $\alpha|0\rangle+\beta|1\rangle$, where $\alpha$ and $\beta$ are complex numbers (numbers of the form $\mathrm{a}+\mathrm{bi}$ ) denoting the corresponding probability that the qubit will collapse to 0 or 1 These coefficients satisfy the equation $|\alpha|^{2}+|\beta|^{2}=1$, because the sum of all probabilities in the system must be 1 . So if the qubit has a $50 \%$ chance of crashing to 0 and a $50 \%$ chance of collapsing to $1, \alpha=1 / \sqrt{2}$ and $\beta=1 /$ $\sqrt{ } 2$.

As seen in Bloch's sphere, $|0\rangle \mathrm{i}|1\rangle$ form the basic vectors of the vector space that describe the state of the qubit. $\alpha$ and $\beta$ can thus be seen as rotations applied to a vector in a complex vector space. We often arrange $\alpha$ and $\beta$ into a column vector known as a quantum state vector, as shown below.

Perhaps even more intriguing is the phenomenon of quantum computation. When two qubits are tangled, the quantum state of one qubit cannot be described independently of the state of the other. Whatever operations are performed with one qubit, the same is true for the other qubit. In reality intertwined quantum particles retain this property even if they are light years apart, which Einstein called "eerie action at a distance."

When one tangled qubit is measured, the superposition of the other qubit also immediately crashes. Thus, instantaneous measurement of the state of a single qubit provides information about the state of a twisted qubit, which makes this specific property incredibly useful.

All of these properties and phenomena are fascinating, but what can be exploited? What makes a quantum computer so revolutionary? There are some specific problems that only quantum computers can solve. The most important and probably the most significant is that they violate modern cryptography. In 1994, Peter Shor showed that a quantum computer could break down globally used encryption algorithms like RSA. RSA supports most of our digital world as it is an integral part of web browsers, messenger applications, email, VPNs.

Quantum computers can be used for quantum simulations, which allows us to visualize and reproduce quantum interactions between molecules and atoms. The consequences are in the fields of quantum chemistry and microbiology. They can improve the speed and performance of machine learning problems and can quickly calculate optimization algorithms that no classic computer has ever been able to.

Quantum computing paves the way for the future of computing, having the potential to revolutionize everything from healthcare to finance. Ultimately, we will all be part of this fantastic revolution.

\section{Conclusion}

If we look briefly at the decision-making process - rational, intuitive, or emotional - understanding the functionality of the human brain is key to improving the quality of human decision-making. We know that neuroinformatics and neurophilosophy are driven by ongoing research in neurobiology, that is, a triangular approach to decision making, integrating all three nodes that need to evolve. To fulfill this task, we need an interdisciplinary platform on which all stakeholders - engineers, neuroscientists, doctors, psychologists, philosophers, educators, legislators communicate to create a networked AI knowledge fund. This, in turn, 
could be the basis for new business models, similar to the Open Source Initiative (OSI) that accelerates the adaptation of information technology through common software modules by reducing costs and time to implement new applications.

Today's global financial situation leads us to conclusions that need to be taken into account through new analyzes not only from a financial point of view but including many scientific branches in order to understand all the complexity of financial decision making.

Monetary policy is never neutral. Namely, a deeper insight shows that monetary policy is always political. "No attempt to map the market structure will ever succeed in removing the political dimension from security purchases. As critical political economists, sociologists and anthropologists so often argue, markets embody a specific, political vision of society, not shared by every policy member. central bank purchases would perfectly reflect the structure of the corporate sector, they would still reflect specific political views".

It is necessary to emphasize stock markets as complex "organisms" of trading. Stock trading algorithms defined sets of instructions based on time, price, quantity, or any mathematical model. In addition to trading opportunities for traders, algo trading makes markets more liquid and more systematic trading by eliminating the impact of human feelings on trading activities. High Frequency Trading (HFT) uses sophisticated algorithms to execute thousands of revolutions per second. High frequency trading, also known as HFT, is a trading method that uses powerful computer programs to transaction a large number of orders in fractions of a second. It uses complex algorithms to analyze multiple markets and execute orders according to market conditions. It is common for traders with the fastest execution speeds to be more profitable than traders with slower execution speeds.

What is the connection of decision-making in financial markets with quantum physics, neuroscience, artificial intelligence?

Neuroscience, both the single-neuron probes and the amazing brain imaging by PET scans and functional MRI, allows us to see (though the pictures are still very blurry and far from the temporal and spatial resolution needed) what is happening in the brain as the mind thinks about various things.

It is unlikely that even the most detailed study of the brain will let us see the indeterministic effects of quantum physics. Looking at a single event is never enough to say that the event was uncaused. Random chance shows up only in the statistics of multiple identical effects.

Economic activity can actively change the brain. Signals predicting regular financial losses cause plastic changes in the crust. Therefore, the brain processes these signals more accurately, which helps to more accurately identify such situations (Scientific Reports, 2020).

The results of the experiment suggest that life economic experience can lead to changes in the brain, which changes the way external signals are perceived. Interestingly, the brain learns to automatically recognize important economic signals. Moreover, scientists have shown precisely how this brain reconnection occurs and have shown the role of individual differences in brain learning systems using the neurotransmitter dopamine.

In a review of the New Neuroscience Literature on the Influences of Moods, Attitudes, and Feelings (Affects) on Financial Decision Making, experiments show us the existence of separate brain systems associated with the influence process responsible for risk behavior and risk avoidance in financial conditions. Excessive activation or suppression of any system can lead to errors in investment selection and trading behavior.

Decisions are the result of complex interactions between many factors. Therefore, it is difficult to determine the importance of different factors in making a final decision. The decisions made by the brain always involve many neurons, so research shows that the weight of individual neurons in the decision-making process can be reconstructed despite the interdependence between neurons. Information enters the brain through many sensory neurons. Which neurons are crucial in passing relevant information to higher areas of the brain? The research team developed an equation that allows them to calculate the degree to which a particular sensory neuron is involved in the decision-making process. To approach this question, the researchers considered information about the final decision made by a particular sensory neuron. Just as an individual is considered suspicious if it is determined to have insider information about a crime, it is assumed that those sensory neurons whose activity contains information about the final decision also played a role in making the final decision. 
The problem with this approach is that neurons - much like humans - are constantly communicating with each other. A neuron that was not involved in the decision itself may have simply received this information from a neighboring neuron and "joined" the conversation. In fact, a neighboring cell transmits a crucial signal that is transmitted to areas of higher decision-making in the brain.

Human beings may have evolved into different types: one whose genetic makeup predisposes them to highrisk and high-return selection, one whose genetic makeup predisposes them to low-risk, low-reward selection. Just as our ancestors chose between hunting and gathering, in part because they had an innate predisposition to risk tolerance or risk aversion, today's people can choose between low-risk and low-risk occupations and high-risk and investment strategies.

Research points to common genetic components of these preferences that lead to correlated behavior among humans. Genetic factors form part of the covariance between domain-specific general risk preferences and risk preferences, between general risk preferences and financial investment choices, between general risk preferences and stock market participation, and between general risk preferences and the choice of enterprise as an occupation. People who are more risk tolerant are more likely to invest in stocks, make riskier financial decisions, and choose risky occupations, in part because of the biological processes underlying their behavior.

Research has also contributed to the biosocial perspective of risk-taking. Domain-specific risk preferences do not have a trivial genetic component. In addition, financial investment selection, entrepreneurship selection, and stock market participation have a significant genetic component. These patterns suggest that crosssectional differences in risk appetite and risk-taking behavior occur naturally in society as a function of genetic composition distribution (Karlsson Linnèr et al., 2019).

Interdisciplinary research with the development of scalar and quantum technologies provides insights into the processes of new financial markets and decision-making systems. This is the foundation of the approach to financial decision making. Quantum technology is a class of technology that operates using the principles of quantum mechanics (subatomic particle physics), including quantum entanglement and quantum superposition. Quantum entanglement and quantum superposition begin to be controlled. This means that quantum technology promises improvements to a wide range of everyday devices, including: a more reliable navigation and timing system, safer communication, more accurate health imaging (quantum reading), and powerful computing. Scalar technology is a product or products that are infused or embedded in scalar waves. It is a unique energy signal that, when properly poured into products, allows them to raise energy more efficiently at the cellular level.

In physics, a scalar is a simple physical quantity that does not change proportions or translations of coordinate systems. The "scalar wave" propagates through dimensional time, but not through dimensional space. Scalar waves generate nonlocal potential energy. They carry information and have a fractal structure. Scalar waves do not decay over time or distance!

\section{References}

1. Aerts, D. (2002). Being and change: Foundations of a realistic operational formalism. In D. Aerts, M. Czachor, \& T. Durt (Eds.), Probing the structure of quantum mechanics: Nonlinearity, nonlocality, probability and axiomatics. Singapore: World Scientific, 71-110. [Link]

2. Aerts, D. (2009). Quantum structures in cognition. Journal of Mathematical Psychology, 53, 314-348. [CrossRef]

3. Aerts, D., \& Aerts, S. (1995). Applications of quantum statistics in psychological studies of decision processes. Foundations of Science, 1, 85-97. [CrossRef]

4. Andersen, R. A. (1995). Encoding of intention and spatial location in the posterior parietal cortex. Cereb. Cortex, 5, 457-469. [CrossRef]

5. Anderson, P. W. (1972). More is different. Science 177, 393-396. [Google Scholar]

6. Aziz R, Verma C, Srivastava N (2018) Artificial neural network classification of high dimensional data with novel optimization approach of dimension reduction. Ann Data Sci 5(4):615-635. [Google Scholar]

7. Blackburn, S. (1999). Think. Oxford: Oxford University Press. ISBN: 9780192100245

8. Bogacz, R., Brown, E., Moehlis, J., Holmes, P., and Cohen, J. D. (2006). The physics of optimal decision making: a formal analysis of models of performance in two-alternative forced-choice tasks. Psychol. Rev. 113, 700-765. [Google Scholar]

9. Bok, H. (1998). Freedom and Responsibility. Princeton: Princeton University Press. [Google Scholar] 
10. Born, R. T., and Bradley, D. C. (2005). Structure and function of visual area MT. Annu. Rev. Neurosci. 28, 157-189. [Google Scholar]

11. Braga AP, Carvalho ACPLF, Ludermir TB (2000) Redes neurais artificiais: teoria e aplicações, 2nd edn. Editora, Rio de Janeiro. [Link]

12. Britten, K. H., Shadlen, M. N., Newsome, W. T., and Movshon, J. A. (1992). The analysis of visual motion: a comparison of neuronal and psychophysical performance. J. Neurosci. 12, 4745-4765. [Google Scholar]

13. Chen, X., Gao, P. (2019). Path planning and control of soccer robot based on genetic algorithm. J Ambient Intell Human Comput. [CrossRef]

14. Chen, JiaWang, et al. (2008). Research on fuzzy control of path tracking for underwater vehicle based on genetic algorithm optimization. Ocean Engineering 156, 217-223. [Google Scholar]

15. Cooper JC (1999) Artificial neural networks versus multivariate statistics: an application from economics. J Appl Stat 26(8):909-921. [CrossRef]

16. Colby, C. L., and Goldberg, M. E. (1999). Space and attention in parietal cortex. Annu. Rev. Neurosci. 22, 319-349. [Google Scholar]

17. Crick, F. (1994). The Astonishing Hypothesis: The Scientific Search for the Soul. New York: Charles Scribner's Sons. [Link]

18. Deaner, R. O., Khera, A. V., and Platt, M. L. (2005). Monkeys pay per view: adaptive valuation of social images by rhesus macaques. Curr. Biol. 15, 543-548. [Google Scholar]

19. Dennett, D. (1984). I could not have done otherwise - so what. J. Philos. 81, 553-567. [Link]

20. Ditterich, J., Mazurek, M., and Shadlen, M. N. (2003). Microstimulation of visual cortex affects the speed of perceptual decisions. Nat. Neurosci. 6, 891-898. [CrossRef]

21. Drugowitsch, J., Moreno-Bote, R., Churchland, A. K., Shadlen, M. N., and Pouget, A. (2012). The cost of accumulating evidence in perceptual decision making. J. Neurosci. 32, 3612-3628. [Google Scholar]

22. Faisal, A. A., Selen, L. P., and Wolpert, D. M. (2008). Noise in the nervous system. Nat. Rev. Neurosci. 9, 292-303. [Google Scholar]

23. Frankfurt, H. G. (1971). Freedom of the will and the concept of a person. J. Philos. 68, 5-21. [Google Scholar]

24. Gazzaniga, M. S. (2011). Who's in Charge? New York: Harper Collins. [Google Scholar]

25. G. Lo Bosco. (2001). A genetic algorithm for image segmentation, Proceedings 11th International Conference on Image Analysis and Processing, Palermo, 262-266.

26. Glimcher, P. (2003). Decisions, Uncertainty, and the Brain: The Science of Neuroeconomics. Cambridge, MA: MIT Press. ISBN 0-262-07244-0. [Google Scholar]

27. Gold, J. I., and Shadlen, M. N. (2002). Banburismus and the brain: decoding the relationship between sensory stimuli, decisions, and reward. Neuron 36, 299-308. [CrossRef]

28. Gold, J. I., and Shadlen, M. N. (2007). The neural basis of decision making. Annu. Rev. Neurosci. 30, 535-574. [Google Scholar]

29. Greene, J., and Cohen, J. (2004). For the law, neuroscience changes nothing and everything. Philos. Trans. R. Soc. Lond. B Biol. Sci. 359, 1775-1785. [Google Scholar]

30. Hanks, T. D., Ditterich, J., and Shadlen, M. N. (2006). Microstimulation of macaque area LIP affects decision-making in a motion discrimination task. Nat. Neurosci. 9, 682-689. [Google Scholar]

31. Hanks, T. D., Kiani, R., and Shadlen, M. N. (2009). A neural correlate of the tradeoff between the speed and accuracy of a decision, in Neuroscience Meeting Planner (Chicago, IL: Society for Neuroscience). [CrossRef]

32. Hanks, T. D., Mazurek, M. E., Kiani, R., Hopp, E., and Shadlen, M. N. (2011). Elapsed decision time affects the weighting of prior probability in a perceptual decision task. J. Neurosci. 31, 6339-6352. [CrossRef]

33. Huang, J., You, X. Y., Liu, H. C., \& Si, S. L. (2019). New approach for quality function deployment based on proportional hesitant fuzzy linguistic term sets and prospect theory. International Journal of Production Research, 57(5), 1283-1299. [Google Scholar]

34. Khashei M, Bijari M (2010) An artificial neural network (p, d, q) model for timeseries forecasting. Expert Syst Appl 37(1):479-489. [CrossRef]

35. Mohammed, Mazin Abed, et al. (2017). Solving vehicle routing problem by using improved genetic algorithm for optimal solution. Journal of computational science, 21, 255-262. [Google Scholar] 
36. Olson DL, Shi Y, Shi Y (2007) Introduction to business data mining, vol 10. McGraw-Hill/Irwin Englewood Cliffs, New York. ISBN 0-02-389340-0. [Google Scholar]

37. Pang, Q., Wang, H., \& Xu, Z. S. (2016). Probabilistic linguistic term sets in multi-attribute group decision making. Information Sciences, 369, 128-143. [CrossRef]

38. Podsiadlo M, Rybinski, H. (2016). Financial time series forecasting using rough sets with time-weighted rule voting. Expert Syst Appl 66:219-233. [Google Scholar]

39. Rath, Asita Kumar, et al. (2019). Path optimization for navigation of a humanoid robot using hybridized fuzzy-genetic algorithm. International Journal of Intelligent Unmanned Systems. [Google Scholar]

40. Ren, P. J., Hao, Z. N., Zeng, X., \& Xu, Z. S. (2020). Decision models based on incomplete hesitant fuzzy linguistic preference relation with application to site section of hydropower stations. IEEE Transcations on Engineering Management. IEEE Transactions on Engineering Management. [Link]

41. Saini, A., Sharma, A. (2019). Predicting the unpredictable: an application of machine learning algorithms in Indian stock market. Ann Data Sci 6, 1-9. [Google Scholar]

42. Shi, Y., Tian Y., Kou, G., Peng, Y., Li, J. (2011). Optimization based data mining: theory and applications. Springer, Berlin. [Google Scholar]

43. Shi, Y. (2014). Big data: history, current status, and challenges going forward. Bridge, 44(4), 6-11. [Google Scholar]

44. Tkáč M, Verner, R. (2016). Artificial neural networks in business: two decades of research. Appl Soft Comput 38:788-804. [CrossRef]

45. Xu, Z., Shi, Y. (2015). Exploring big data analysis: fundamental scientific problems. Ann Data Sci, 2(4), 363-372. [Google Scholar]

46. Wang, J., et al. (2016). Multi-offspring genetic algorithm and its application to the traveling salesman problem. Applied Soft Computing 43 415-423. [CroosRef] 